Please do not remove this page

RMIT

UNIVERSITY

\title{
Alterations in human EEG activity caused by extremely low frequency electromagnetic fields
}

Cvetkovic, Dean; Jovanov, Emil; Cosic, Irena

https://researchrepository.rmit.edu.au/esploro/outputs/9921862791401341/filesAndLinks?institution=61RMIT_INST\&index=null

Cvetkovic, D., Jovanov, E., \& Cosic, I. (2006). Alterations in human EEG activity caused by extremely low frequency electromagnetic fields. 28th IEEE Engineering in Medicine and Biology Society (EMBS) Annual International Conference, 3206-3209. https://doi.org/10.1109/IEMBS.2006.259314

Published Version: https://doi.org/10.1109/IEMBS.2006.259314

Repository homepage: https://researchrepository.rmit.edu.au

(c) 2006 IEEE. Personal use of this material is permitted. However, permission to reprint/republish this material for advertising or promotional purposes or for creating new collective works for resale or redistribution to servers or lists, or to reuse any copyrighted component of this work in other works must be obtained from the IEEE.

Downloaded On 2023/04/26 17:41:47+1000 


\title{
Alterations in Human EEG Activity Caused by Extremely Low Frequency Electromagnetic Fields
}

\author{
D. Cvetkovic, E. Jovanov, Senior Member, and I. Cosic, Senior Member
}

\begin{abstract}
This study has investigated whether extremely low frequency (ELF) electromagnetic fields (EMFs) can alter human brain activity. Linearly polarised magnetic flux density of $20 \mu \mathrm{T}$ (rms) was generated using a standard double Helmholtz coils and applied to the human head over a sequence of 1 minute stimulations followed by one minute without stimulation in the following order of frequencies 50, 16.66, 13, $10,8.33$ and $4 \mathrm{~Hz}$. We collected recordings on 33 human volunteers under double-blind counter-balanced conditions. Each stimulation lasted for two minutes followed by one minute post-stimulation EEG recording. The same procedure was repeated for the EMF control sessions, where the order of control and exposure sessions was determined randomly according to the subject's ID number. The rest period between two conditions (exposure and control) was 30 minutes. The results indicate that there was a significant increase in Alpha1, Alpha2, and Beta1 at the frontal brain region, and a significant decrease in Alpha2 band in parietal and occipital region due to EMF exposure.
\end{abstract}

\section{INTRODUCTION}

$\mathrm{S}_{\mathrm{e}}^{\mathrm{e}}$ everal studies have been conducted to asses whether electromagnetic field (EMF) exposures at characteristic frequencies of brain electrical activity could influence alterations in the EEG and other physiological parameters. Studies on $16.66 \mathrm{~Hz}$ and $50 \mathrm{~Hz}$ have reported adverse effects on humans and animals [1]-[4]. A single-blind study on 61 volunteers exposed to alternating $3 \mathrm{~Hz}$ magnetic field of $0.1 \mathrm{mT}$ for 20 minutes caused relative spectral power increase at theta and alpha EEG bands and decrease in beta EEG band at the occipital head regions [5]. It was previously reported that applications of electromagnetic fields (EMFs) in the range $0-60 \mathrm{~Hz}$ and intensity $20-100 \mu \mathrm{T}$, altered EEG activity in animals and human subjects during 2-second exposure epochs [6]. It was concluded that a weak EMF applied continuously to human subjects for 10 minutes resulted in a reduction in brain electrical activity at the

Manuscript received April 3, 2006

D. Cvetkovic (corresponding author) is with Australian Centre for Radiofrequency Bioeffects Research (ACRBR) and RMIT University, School of Electrical and Computer Engineering, GPO Box 2476V, Melbourne, VIC 3001, Australia, tel: +613 9925 9641, fax: +6139925 2007, e-mail: dean.cvetkovic@rmit.edu.au.

E. Jovanov is with Electrical and Computer Engineering Dept, University of Alabama in Huntsville, Huntsville, Alabama 35899, U.S.A. tel: +1 (256) 824 5094, fax: +1 (256) 824 6803, e-mail: jovanov@ece.uah.edu.

I. Cosic is with Australian Centre for Radiofrequency Bioeffects Research (ACRBR) and RMIT University, School of Electrical and Computer Engineering, GPO Box 2476V, Melbourne, VIC 3001, Australia, tel: +613 9925 1971, fax: +613 9925 2007, e-mail: irena.cosic@ rmit.edu.au. frequency of the EMF during the 1-minute interval following termination of the field. A similar study reported on the

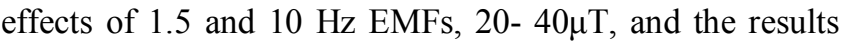
indicated altered brain EEG activity [7]. A recent double blind study on 20 subjects suggested that exposure to ELF magnetic fields altered human EEG activity, specifically within the alpha frequency band $(8-13 \mathrm{~Hz})[8]$. The findings indicated that alpha activity was significantly higher over the occipital electrodes and marginally higher over the parietal electrodes at post-exposure.

The purpose of this study was to investigate whether combined extremely low frequencies EMFs (50, 16.66, 13, $10,8.33$ and $4 \mathrm{~Hz}$, in that order) which have previously been investigated in literature only as individual frequencies, could cause changes in the EEG activity under double-blind and counter-balanced conditions.

\section{MATERIALS AND METHODS}

\section{A. Design of Helmholtz Coil Magnetic Field Exposure Apparatus}

The preliminary [9],[10] and final studies investigated whether multiple sinusoidal extremely low frequency (ELF) $(50,16.66,13,10,8.33$ and $4 \mathrm{~Hz})$ linearly polarised magnetic flux density of $20 \pm 0.57 \mu \mathrm{T}$ (rms) applied to the human head over a non-continuous period of 12 minute, could causes alterations in the EEG rhythms on 33 human volunteers [11]. Standard circular Helmholtz pair of coils have been designed to pass the current of approximately $140 \mathrm{~mA}$. The total coil impedance was $71 \Omega$, designed with average radii of $65 \mathrm{~cm}$, copper wire of $0.8 \mathrm{~mm}$ in diameter and 250 turns each. A signal generator effective in producing high quality sine waveforms of high stability/accuracy ELF signals was designed and developed using EXAR XR-2206 monolithic IC. Also, an audio amplifier was designed and constructed with the approximate gain of 10 to deliver sufficient current to the coils. The magnetic flux density was verified by direct measurement using "Wandel and Goltermann" EFA-200 EMF Analyser. The linearly polarized filed was perpendicular to the Earth's North-South magnetic field.

\section{B. Subjects and EEG Montages and Procedures}

The final experiments were conducted on 33 healthy subjects, 24 male and 9 female, with mean age of 30 years, SD 11 years, range 20-59 years. The RMIT ethics committee approved the study and all subjects gave written informed 
consent prior to the experiment. During the EEG recording sessions, subjects were asked to lie down between the coils in saggital plane direction perpendicular to the coil axis and in the supine position. The entire experiment was performed in a darkened and sound proof RF anechoic chamber to prevent erroneous recordings due to the standing waves and power line interference.

\section{EEG Recording and Experimental Protocol}

The EEG equipment used throughout testing was the Mindset MS-1000 recording system. Neuroscan 19 Channel Caps electrodes were used with referential montage of 16 channels. The left brain hemisphere electrodes: Fp1, F7, F3, T7, C3, P7, P3 and O1 were all referenced to M1 (left mastoid), while the right brain hemisphere electrodes: Fp2, F8, F4, T8, C4, P8, P4 and O2 were referenced to right mastoid M2. The baseline EEG was recorded prior to any stimulation for one minute. Each stimulation (50, 16.66, 13, $10,8.33$ and $4 \mathrm{~Hz}$ ) lasted for two minutes followed by one minute post-stimulation EEG recording. Therefore, total length of an experiment was 19 minutes. The same procedure was repeated for the EMF control sessions. The order of control and exposure sessions was determined randomly according to the subject's ID number. Subjects with odd ID numbers were first tested with control condition (no EMF exposure) followed by EMF stimulation after 30 minute break. Double-blind counterbalanced condition was exercised. The two EMF sessions were highly considered in the analysis as a factor that might reveal that if the $1^{\text {st }}$ session was EMF exposure, the EEG activity results during the $2^{\text {nd }}$ EMF control session could still be influenced or dependent on the results of the $1^{\text {st }}$ EMF exposure session.

\section{Signal Processing and Statistical Method}

All the collected EEG data was processed using Matlab tool. The main Matlab script was written to process all 16 channel EEG data of all subjects and generate valuable parameters that would be used in the further statistical analysis, such as Total spectral power of each stimulation EEG data (i.e. before, $50 \mathrm{~Hz}, 16.66 \mathrm{~Hz}, 13 \mathrm{~Hz}, 10 \mathrm{~Hz}, 8.33 \mathrm{~Hz}$ and $4 \mathrm{~Hz}$; Spectral power in the stimulated band, before/after; Central band frequency before/after and Relative difference "ratio" between the individual band and total spectral power before/after. Spectral function was written to compute the windowed discrete-time Fourier transform of a signal using a sliding window. The EEG band intervals were as Theta (3-5Hz), Alpha1 (7.5-9.5Hz), Alpha2 $(9-11 \mathrm{~Hz})$, Beta1 $(12-14 \mathrm{~Hz})$, Beta2 $(15.5-17.5 \mathrm{~Hz})$ and Gamma $(49-51 \mathrm{~Hz})$. Delta and Gamma band data was excluded from this particular analysis. We compared the EEG activity "before" and "after" stimulation for each frequency stimulation and band. Throughout this method, "before" stimulation EEG data was regarded for every next recording of the "after". For example, if $1^{\text {st }}$ recording was before any stimulation, $2^{\text {nd }}$ was $50 \mathrm{~Hz}$ stimulation (gamma band), $3^{\text {rd }}$ was $16.66 \mathrm{~Hz}$ stimulation (beta2 band). The script used for this signal processing computed all the parameters mentioned above as 1 second epochs, maximum of 60 epochs per recording. Throughout this investigation, only the relative difference (ratio) parameter between the individual bands and total spectral power (before and after) was used for the statistical analysis.

\section{RESULtS}

Multiple paired samples 2-tailed t-tests and ANOVA's 3-way mixed design for within and between-subject measures were employed. The factors considered were the "before and after", "exposure and control" and "first and second session." The first test conducted was for the first session of EMF exposure and there were 16 subjects used for this session. The second test was the second session EMF control $(d f=15)$, the third test was the first session EMF control $(d f=16)$ and the fourth test was the second session EMF exposure $(d f=16)$.

\section{A. EMF Exposure followed by EMF Control Results}

In Alphal band and $8.33 \mathrm{~Hz}$ stimulation under EMF control ( $2^{\text {nd }}$ session), $t$-test results revealed a significant relative difference increase from before to after at $\mathrm{T} 7(t(15)=$ -2.397, $\mathrm{p}<0.030)$. ANOVA test revealed a significant difference for the interaction between exposure/control and sessions factors $(\mathrm{T} 7)(\mathrm{F}(1,31)=5.992, \mathrm{p}<0.020)$. In Alpha2 band after $10 \mathrm{~Hz}$ stimulation, $2^{\text {nd }}$ control session, the relative difference has decreased, highlighted by a high difference observed in parietal and occipital regions, P3, that the relative difference at before $(\mathrm{M}=0.1789, \mathrm{SE}=0.0201)$ was significantly higher than after $(\mathrm{M}=0.1573, \mathrm{SE}=0.0140)$, $\mathrm{t}(15)=3.081, \mathrm{p}<0.008$. At $\mathrm{P} 4$, the relative difference before $(\mathrm{M}=0.1861, \mathrm{SE}=0.0223)$ was significantly higher than after $(\mathrm{M}=0.1510, \quad \mathrm{SE}=0.0134), \mathrm{t}(15)=2.812, \quad \mathrm{p}<0.013$. The occipital regions, $\mathrm{O} 1$ before $(\mathrm{M}=0.1399, \mathrm{SE}=0.0156)$ and after $(\mathrm{M}=0.1243, \mathrm{SE}=0.0111), \mathrm{t}(15)=2.256, \mathrm{p}<0.039$; and $\mathrm{O} 2$ before $(\mathrm{M}=0.1383, \mathrm{SE}=0.0137)$ and after $(\mathrm{M}=0.1203$, $\mathrm{SE}=0.0104), \mathrm{t}(15)=3.283, \mathrm{p}<0.005$, as shown in Figure 1 . There was a largest decrease in relative difference from before to after by $12 \%(\mathrm{P} 3), 18.4 \%(\mathrm{P} 4), 11.2 \%(\mathrm{O} 1)$, and $13 \%(\mathrm{O} 2)$ than at any other electrode and stimulation. The 3-way ANOVA revealed a significant difference at the interaction between exposure/control and sessions (P3) $\mathrm{F}(1,31)=11.918, \mathrm{p}<0.002$ and the main factor before/ after $\mathrm{F}(1,31)=5.230, \mathrm{p}<0.029$. At $\mathrm{P} 4$ electrode, a significant difference between exposure/control and sessions was $\mathrm{F}(1,31)=14.827, \mathrm{p}<0.001$ and before/after $\mathrm{F}(1,31)=4.406$, $\mathrm{p}<0.044 ; \quad \mathrm{O} 1 \quad$ revealed $\mathrm{F}(1,31)=9.346, \quad \mathrm{p}<0.005$ (exposure/control and sessions); and $\mathrm{O} 2 \mathrm{~F}(1,31)=13.071$, $\mathrm{p}<0.001$. The t-test results for $13 \mathrm{~Hz}$ stimulation in Beta 1 band revealed no significant differences at any electrode, as shown in Figure 2. 


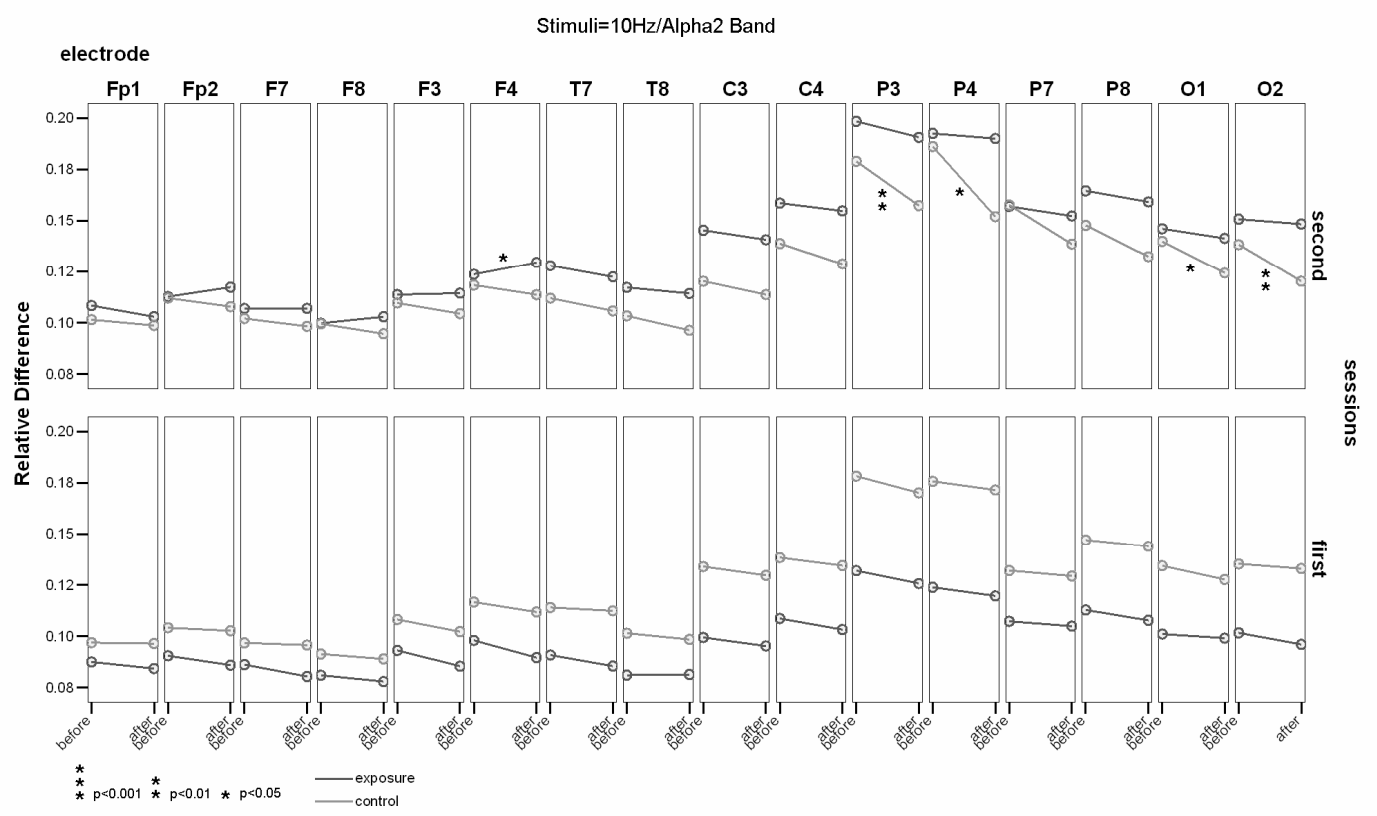

Figure 1. The Relative Differences Versus "Before" and "After" Results Represented at 10Hz Stimulation in a Alpha2 band for EMF Exposure/Control and First/Second Session Conditions.

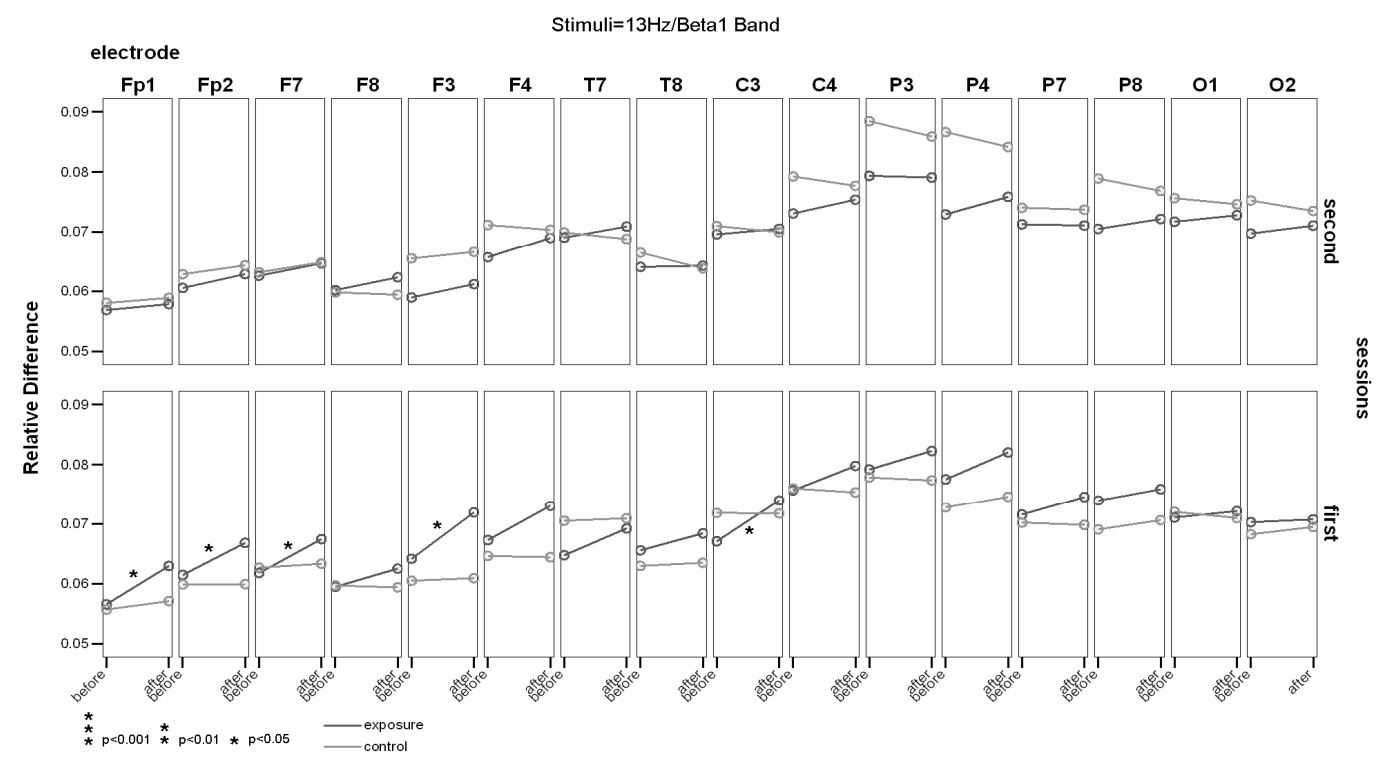

Figure 2. The Relative Differences Versus "Before" and "After" Results Represented at 13Hz Stimulation in a Beta1 band for EMF Exposure/Control and First/Second Session Conditions.

For the $1^{\text {st }}$ EMF exposure session, the t-test results revealed a significant increase at Fp1, Fp2, F7, F3 and C3 for $13 \mathrm{~Hz}$ stimulation in Beta1 band. At F7 before, $\mathrm{t}(1,15)=-2.798$, $\mathrm{p}<0.014 ; \mathrm{F} 3$ before $\mathrm{t}(1,15)=-2.659, \mathrm{p}<0.018$; and $\mathrm{C} 3$ before $\mathrm{t}(1,15)=-2.391, \mathrm{p}<0.030$. There was an increase in relative difference from before to after by $10.1 \%(\mathrm{Fp} 1), 8 \%(\mathrm{Fp} 2)$, $8.4 \%$ (F7), 10.8\% (F3) and 9.3\% (C3). The ANOVA results revealed a significant differences between before and after main factors at $F p 1 F(1,31)=12.852, p<0.001 ; F p 2 F(1,31)=$ 7.058, $\mathrm{p}<0.012$; F7 $\mathrm{F}(1,31)=15.730, \mathrm{p}<0.0001$; and $\mathrm{C} 3$ (NS). In $1^{\text {st }}$ EMF exposure Betal band (13Hz), ANOVA's significant results for before and after main factor, were very similar with the t-test's results.

\section{B. EMF Control followed by EMF Exposure Results}

For the $2^{\text {nd }}$ EMF exposure session, the t-tests were conducted for $8.33 \mathrm{~Hz}$ stimulation in Alphal band, that relative difference at electrodes Fp1, F7, F3, F4 and C4 was significantly higher before than after stimulation. The results of t-tests were: $\mathrm{F} 7$ before $\mathrm{t}(1,16)=2.120, \mathrm{p}<0.050 ; \mathrm{F} 3$ before $\mathrm{t}(1,16)=2.862, \mathrm{p}<0.011 ; \mathrm{F} 4$ before $\mathrm{t}(1,16)=2.682, \mathrm{p}<0.016$; and $\mathrm{C} 4$ before $\mathrm{t}(1,16)=2.872, \mathrm{p}<0.011$. There was a decrease 
in relative difference from before to after by $11.1 \%$ (Fp1), $11.3 \%$ (F7), 10\% (F3), 9.8\% (F4) and 8.8\% (C4). The ANOVA results indicated a significant difference at: F7 $\mathrm{F}(1,31)=6.485, \mathrm{p}<0.016$ (exposure/control and sessions) and $\mathrm{F}(1,31)=4.485, \mathrm{p}<0.042$ (before/after and sessions); $\mathrm{F} 3$ $\mathrm{F}(1,31)=4.524, \mathrm{p}<0.041$ (exposure/control and sessions) and $\mathrm{F}(1,31)=4.297, \mathrm{p}<0.047$ (before/after and sessions); $\mathrm{F} 4$ $\mathrm{F}(1,31)=11.554, \mathrm{p}<0.002$ (exposure/control and sessions); and $\mathrm{C} 4 \mathrm{~F}(1,31)=5.121, \mathrm{p}<0.031$ (exposure/control and sessions) and $\mathrm{F}(1,31)=6.035, \mathrm{p}<0.020$ (before/after and sessions). Under the $2^{\text {nd }}$ EMF exposure session, the t-test revealed a significant difference between before and after stimulation of $10 \mathrm{~Hz}$ in Alpha2 band at F4, where a relative difference was higher before than after the $10 \mathrm{~Hz}$ stimulation $\mathrm{t}(16)=-2.130, \mathrm{p}<0.049$, as shown in Figure 1. ANOVA revealed a significant difference for the interaction between exposure/control and session's factor, $\mathrm{F}(1,31)=11.043$, $\mathrm{p}<0.002$. For $13 \mathrm{~Hz}$ stimulation, there was no significant difference.

\section{DISCUSSION}

The statistical EMF exposure/control tests have been conducted and the summary of its entire hypothesis tested have been illustrated in Figure 3.

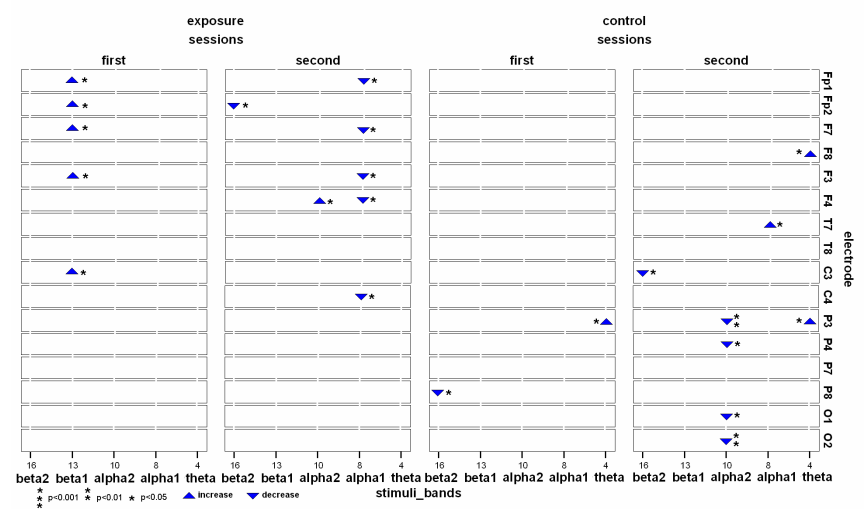

Figure 3. Summary of All the T-test Significant Relative Increase/Decrease for the Standard EMF Exposure/Control and First/Second Session Conditions at Individual Bands/Stimuli and Electrodes.

The alternative hypothesis test for EMF Exposure $1^{\text {st }}$ and Control $2^{\text {nd }}$ session results signify a possibility that the EEG activity could remain altered for at least 50 minutes after the exposure (30 minutes break between the exposure and control conditions with additional 20 minutes for EMF control EEG recordings and stimulations). For the corrected alpha rate value of multiple tests, Bonferroni test was used with the new modified alpha rate of $p<0.0025$. No significant differences were observed as a result of this correction. However, the final analysis results suggest that EEG activity in Alpha1, Alpha2 and Beta1 band could be altered due to EMF exposures, which are mainly associated with stimulation frequencies of $8.33,10$ and $13 \mathrm{~Hz}$.

\section{CONCLUSION}

The results from the EEG study on 33 subjects have indicated that under the first EMF exposure there was a shift from a significant increase in Betal band at frontal region of the brain to a significant decrease in Alpha2 band at the back region (parietal and occipital) under the post-EMF exposure. However, when the subjects were exposed to EMF after 60 minutes of rest, they exhibited a decrease in Alphal band.

\section{ACKNOWLEDGMENT}

The authors gratefully acknowledge the NHMRC for their support of the ACRBR which has assisted this research work.

\section{REFERENCES}

[1] H. Brendel, M. Niehaus, A. Lerchl, "Direct supressive effects of weak magnetic fields $(50 \mathrm{~Hz}$ and $16.66 \mathrm{~Hz})$ on melatonin synthesis in the pineal gland of Djungarian hamsters (Phodopus sungorus)", Journal of Pineal Research, vol. 29, pp. 228-233, 2000.

[2] M.J. Azanza, A.C. Calvo, A.D. Moral, "Evidence of synchronisation of neural activity of molluscan brain ganglia induced by alternating $50 \mathrm{~Hz}$ applied magnetic field", in Electromagnetic Biology and Medicine, Electromagnetic Biology and Medicine, 2002.

[3] T. Akerstedt, B. Arnetz, G. Ficca, L.E. Paulsson, A. Kallner, “A 50Hz Electromagnetic field impairs sleep", Journal of Sleep Research, vol. 8, pp. 77-81, 1999.

[4] Y. Kurokawa, H. Nitta, H. Imai, M. Kabuto, “Acute Exposure to $50 \mathrm{~Hz}$ Magnetic Fields with Harmonics and Transient Components: lack of effects on nighttime hormonal secretion in men", Bioelectromagnetics, vol. 24, pp. 12-20, 2003.

[5] K. Hausser, D. Tellschaft, F. Thoss, "Influence of an Alternating $3 \mathrm{~Hz}$ Magnetic Field with an Induction of $0.1 \mathrm{mT}$ on Chosen Parameters of the Human Occipital EEG", Neuroscience Letters, vol. 239, pp. 57-60, 1997.

[6] G.B. Bell, A.A. Marino, A. Chesson, "Frequency-Specific Blocking in the Human Brain Caused by Electromagnetic Fields", Neuro Report, vol. 5, pp. 510-512, 1994.

[7] G.B. Bell, A.A. Marino, A. Chesson, "Frequency-Specific Responses in the Human Brain Caused by Electromagnetic Fields", Journal of the Neurological Sciences, vol. 123, pp. 26-32, 1994.

[8] C.M. Cook, A.W. Thomas, F.S. Prato, "Resting EEG is affected by exposure to a pulsed ELF magnetic field". Bioelectromagnetics, vol. 25: pp. 196-203, 2004.

[9] D. Cvetkovic, I. Cosic, "The induced rhythmic oscillations of neural activity in the human brain" in Proc. IASTED (BIOMED) Conf., ACT A Press, 2004.

[10] D. Cvetkovic, I. Cosic, "Automated ELF Magnetic Field Stimulation on the Human EEG Activity", Integrated Computer-Aided Engineering, IOS Press, 2006 (accepted).

[11] D. Cvetkovic, "Electromagnetic and Audio-Visual Stimulation of the Human Brain at Extremely Low Frequencies" Ph.D. dissertation, School of Electrical and Computer Engineering, RMIT Univ., Melbourne, Australia, 2005. 